\title{
Philosophiques
}

\section{Freud et la guerre}

\section{David Cumin}

Volume 35, numéro 2, automne 2008

URI : https://id.erudit.org/iderudit/000435ar

DOI : https://doi.org/10.7202/000435ar

Aller au sommaire du numéro

Éditeur(s)

Société de philosophie du Québec

ISSN

0316-2923 (imprimé)

1492-1391 (numérique)

Découvrir la revue

Citer cet article

Cumin, D. (2008). Freud et la guerre. Philosophiques, 35(2), 393-417.

https://doi.org/10.7202/000435ar

Ce document est protégé par la loi sur le droit d'auteur. L'utilisation des services d'Érudit (y compris la reproduction) est assujettie à sa politique d'utilisation que vous pouvez consulter en ligne.

https://apropos.erudit.org/fr/usagers/politique-dutilisation/
Cet article est diffusé et préservé par Érudit.

Érudit est un consortium interuniversitaire sans but lucratif composé de l’Université de Montréal, l'Université Laval et l'Université du Québec à Montréal. Il a pour mission la promotion et la valorisation de la recherche. https://www.erudit.org/fr/ 


\title{
Freud et la guerre*
}

\author{
DAVID CUMIN \\ Maître de conférences (HDR) à l'Université Jean Moulin, Lyon III (CLESID)
}

La psychologie était une branche de la philosophie, jusqu'à ce que Sigmund Freud (1856-1939) invente la méthode de recherche que l'on nomme psychanalyse. La psychanalyse est un procédé d'investigation des processus psychiques, une théorie de la psyché, une méthode de traitement des désordres psychiques. Son édifice théorique repose sur un corps de doctrines : refoulement, inconscient, complexes, signification étiologique de la vie sexuelle, importance des expériences vécues dans l'enfance, interprétation des rêves... La psychanalyse consiste en l'examen de la partie inconsciente de la vie psychique ${ }^{1}$, afin d'expliquer ce qui paraît inexplicable dans les conduites humaines. En tant que phénomène multidimensionnel, la guerre intéresse évidemment la psychologie; Gaston Bouthoul, fondateur de la polémologie en France², le soulignait. Que dit Freud, savant, sur la guerre? D’emblée, il place le conflit au centre de la psychologie humaine : conflit du ça et du surmoi, de la pulsion de vie (Eros) et de la pulsion de mort (Thanatos), du père et du fils. Il a pourtant peu écrit sur la guerre stricto sensu: conflit armé entre belligérants. Il a publié une centaine de textes: articles, contributions, livres. Une demidouzaine, portant notamment sur la religion, intéressent la question de la guerre. Mais deux seulement lui sont explicitement et directement consacrés : "Considérations actuelles sur la guerre et sur la mort », rédigé en 1915 (30 pages); «Pourquoi la guerre? », rédigé en 1933 (10 pages). La Première Guerre mondiale avait pourtant accru l'intérêt pour la psychanalyse d'un point de vue clinique : l'observation et la guérison des névrosés de guerre. La réflexion

*Cf. dans la bibliographie les références des ouvrages et articles cités.

1. Totem et tabou (1913), p.45.

2. Gaston Bouthoul (1896-1980), docteur en droit et en philosophie, a été, entre autres, professeur à l'École des hautes études en sciences sociales. Il a fondé la polémologie, c'est-à-dire l'étude scientifique des conflits armés, et l'Institut français de polémologie, lequel a accumulé une considérable masse documentaire : de 1945 à 1993, tous les phénomènes de violence politique y ont été recensés, analysés, classés. L'œuvre, partiellement traduite à l'étranger, de Bouthoul est immense. On doit citer, publiés à Paris : Cent millions de morts, Sagittaire, 1946; Huit mille traités de paix, Julliard, 1948 ; Les guerres. Eléments de polémologie, Payot, 1951; La guerre, PUF, QSJ, 1953, 1963 ; Sauver la guerre, Grasset, 1961 ; Traité de polémologie, Payot, 1951, 1961, 1970 ; Le phénomène guerre, Payot, 1962; Avoir la paix, Grasset, 1967; L'infanticide différé, Hachette, 1970 ; Lettre ouverte aux pacifistes, A. Michel, 1972; Le défi de la guerre (1740-1974). Deux siècles de guerres et de révolutions, PUF, 1976 (avec le général René Carrère); Essais de polémologie, Denoël/Gonthier, 1976; Guerres et civilisations, FEDN, 1979 (avec René Carrère et Jean-Louis Annequin). S'ajoutent de nombreux ouvrages de démographie, sociologie, histoire des idées. Mentionnons également les deux revues de l'IFP : Guerres et Paix (11 numéros de 1966 à 1970) puis Études polémologiques (52 numéros de 1971 à 1990) dans lesquelles Bouthoul publia de nombreux articles (Cultures et Conflits a succédé à Études polémologiques en 1991). 
freudienne n'en est pas moins importante et étonnante. Nombre de travaux sur la guerre ont pour origine le pacifisme: "Tout pacifisme conséquent appelle un examen des racines de la guerre » (Alain). Est-ce le cas chez notre auteur ? L'objectif de cet article est de démontrer, textes à l'appui, que Freud ne croyait pas au pacifisme, du moins pas au pacifisme juridique.

\section{La position politique de Freud}

Il convient de situer politiquement le personnage. On a souvent rapproché Freud de Marx: deux auteurs germaniques, d'origine juive, qui se réfugièrent à Londres ${ }^{3}$, l'œuvre du premier étant volontiers considéré comme le pendant psychanalytique de l'œuvre économique du second. Le retour aux sources documentaires, autrement dit, la lecture de Freud dans le texte, autorise à corriger l'interprétation «de gauche ${ }^{4}$. En science du droit, le kelsénisme n'est pas $K^{K e l s e n}{ }^{5}$; en psychologie, le freudisme n'est peut-être pas Freud! Dans le rapport Freud/Marx, on insiste sur l'athéisme, le caractère "révolutionnaire » de la psychanalyse (son fondateur en soulignait pourtant la "neutralité éthique »), la critique sociale et le projet émancipatoire : éduquer et libérer l'homme de ses aliénations psychologiques. Certes. On sait pourtant à quel point le dépassement du rationalisme et de l'individualisme classiques doit à Freud. Celui-ci était un rationaliste dans le sens des Lumières; l'originalité de son œuvre a cependant consisté, non à fonder la supériorité de la raison, mais à restituer aux ratés et aux illogismes de l'activité mentale: lapsus, oublis, erreurs, rêves..., leur force de vérité.

La lecture des ouvrages du savant révèle les traits suivants: pessimisme anthropologique et culturel; hiérarchisation des peuples civilisés et des peuples primitifs; croyance en l'hérédité ( « nos actes conscients dérivent d'un substrat inconscient formé surtout d'influences héréditaires » $)^{6}$ élitisme ; conviction que l'éducabilité des masses est limitée, qu'elles doivent être contraintes à se conformer aux règles et que le pouvoir est par conséquent nécessaire; scepticisme sur le "progrès ${ }^{7}$; conception patriarcale de la société et de la famille ( « le passage de la mère au père caractérise une victoire de la vie

3. Mais Freud vécut presque toute sa vie à Vienne (jusqu'en 1938) et il officia 42 ans dans son cabinet viennois!

4. Dans le $n^{\circ} 12 / 13$ de la revue Aufstieg, Paul Federn publia en 1919, à Vienne, sa «Contribution à la psychologie de la révolution: la société sans père ». La révolution vise la substitution de la société patriarcale, symbolisée par l'État et l'Église, par une "société de frères égaux ». Exemple d'une interprétation de Freud... que Freud n'aurait certainement pas approuvée! Cf. H. Glaser, Sigmund Freud et l'âme du XX $X^{e}$ siècle, pp. 212-215, 391-392. 1997.

5. Cf. Carlos Miguel Herrera: Théorie juridique et politique chez Hans Kelsen, Paris, Kimé,

6. "Psychologie des foules et analyse du moi » (1921), in Essais de psychanalyse, p. 128.

7. Dans la postérité freudienne, l'hypothèse de la pulsion de mort, d'emblée fort mal accueillie, constitua une véritable pomme de discorde: si l'homme est destructeur en soi, pourquoi lutter pour un avenir meilleur? 
de l'esprit sur la vie des sens, donc un progrès de la civilisation » $)^{8}$; attirance pour les religions ( «tout ce qui a trait à l'origine d'une religion possède en soi quelque chose de grandiose $»)^{9}$ et les mythes (la «psychologie des profondeurs "); intérêt pour les figures charismatiques ${ }^{10}$, Moïse, qu'il admire, ou Wilson, qu'il méprise; préférence pour la «culture » par opposition à la "civilisation » ${ }^{11}$, récusation de l'anti-germanisme et défense des Empires cen$\operatorname{traux}^{12}$ contre la propagande alliée ${ }^{13}$; critique du Traité de Versailles ("véritable condamnation à mort de la civilisation européenne $»)^{14}$ et du Pacte de la SDN ; anticommunisme ${ }^{15}$ et anti-américanisme; "réalisme» en relations internationales, comme l'illustre son extraordinaire critique du wilsonisme, dans le fameux Portrait psychologique du Président américain... Après 1933, c'est dans l'Église catholique, pas dans la sociale-démocratie, que Freud voit le meilleur rempart contre le fascisme ${ }^{16}$.

8. Moïse (1938), p. 213. En cela, Freud s'opposait à Otto Gross ou à Wilhelm Reich.

9. Idem, p. 231.

10. «Il est impossible de nier l'influence personnelle des grands hommes sur l'histoire universelle. " «Le grand homme influence ses contemporains de deux manières : par sa personnalité et par l'idée pour laquelle il s'engage. " "Il existe dans la masse le besoin d'une autorité que l'on puisse admirer. " Avec Moïse, "la figure du grand homme s'est amplifiée jusqu'au divin " (Moïse, pp. 128, 207, 208). "Tout le cours de la vie peut être détourné par le tempérament d'un seul individu. Quand Wilson quitta Paris, le cours de la civilisation occidentale prit une direction pénible à voir. L'humanité a besoin de héros, et de même que celui qui est fidèle à son devoir élève le niveau de la vie humaine, celui qui le trahit l'abaisse. Wilson parla merveilleusement, fit de superbes promesses, puis capitula»(Wilson, 1938, p. 289).

11. Freud parle de Thomas Mann comme «l'un des auteurs qui avait le plus vocation à être le porte-parole du peuple allemand » : l'auteur des Considérations d'un apolitique, défenseur de la Kultur germanique face à la Zivilisation occidentale.

12. «Lorsque, pendant la guerre mondiale, un chœur d'ennemis lança contre la nation allemande le reproche de barbarie, cela me peina douloureusement de ne pouvoir y apporter le démenti de ma propre expérience» (Sigmund Freud présenté par lui-même, 1935, 1925, p. 83). En 1915, il écrit : « une des grandes nations civilisées est si généralement détestée qu'on peut être tenté de l'exclure, en tant que 'barbare', de la communauté civilisée, bien qu'elle ait prouvé par les contributions les plus grandioses son aptitude à en faire partie. Nous vivons dans l'espoir qu'une version impartiale de l'histoire apportera la preuve que cette nation justement, celle dans la langue de laquelle nous écrivons, pour la victoire de laquelle combattent ceux qui nous sont chers, est celle qui a le moins violé les lois de la morale humaine » ("Considérations actuelles sur la guerre et la mort ", 1915, in Essais de psychanalyse, p. 14).

13. Jusqu'en 1938. C'est Wilson dont il brosse la psychologie critique ou la critique psychologique, dans un ouvrage qui est une véritable mine de renseignements sur la position et l'évolution de la diplomatie américaine de 1914 à 1919.

14. Wilson, p. 328.

15. Freud dira avec ironie que la rencontre avec un militant l'avait à moitié converti. Ce dernier affirmait que l'avènement du bolchevisme amènerait quelques années de misère et de chaos, mais qu'elles seraient suivies de la paix universelle. Il lui répondit qu'il croyait à la première moitié du programme (cité par E. Jones, La vie et l'œuvre de Sigmund Freud, t. III, Paris, PUF, 1969, p. 18). Cf. les pages 55-57 du Malaise dans la culture (1930).

16. Avant mars 1938, date de l'Anschluss, il écrit dans Mö̈se: "nous vivons ici dans un pays catholique sous la protection de cette Église » (pp. 132, 135). 
Politiquement, Freud était peut-être social-démocrate. Culturellement, il était assurément un grand conservateur européen de son temps. Un « représentant de l'idéologie puritaine bourgeoise », quelqu'un qui n'a jamais cessé «de reconnaître la nécessité de la convention et de l'institution" (H. Glaser), qui était fier de «notre culture blanche et chrétienne contemporaine ${ }^{17}$. Il était un athée qui soulignait et redoutait le danger social de nier Dieu, pour qui la principale menace pesant sur la culture était la révolte pulsionnelle des masses, pour qui l'antisémitisme hitlérien n'était au fond qu'un antichristianisme ${ }^{18}$. Face à la Russie soviétique, à l'Italie fasciste et à l'Allemagne nationale-socialiste, les démocraties conservatrices lui semblaient les gardiennes du progrès culturel ${ }^{19}$. À l'encontre d'Alfred Adler, il ne voyait entre le marxisme et la psychanalyse aucune filiation. À l'inverse du matérialisme de Marx, Freud part de la conscience ${ }^{20}$. En revanche, il reconnaissait, outre la référence à Darwin, la convergence de la psychanalyse avec les philosophies de Schopenhauer et de Nietzsche. Étudiant à l'Université, il avait adhéré à une association pangermaniste. Ses biographes observent qu'il votait peu et méprisait volontiers les politiciens de l'époque. Il aimait à se présenter comme un « opposant $»^{21}-$ mais à quoi ?

\section{L'interdit de l'homicide et sa transgression}

Les textes intéressant la question de la guerre paraissent entre 1913 et 1938, alors que leur auteur est parvenu à la notoriété dans son domaine médical. Il s'agit donc de l'œuvre d'un homme mûr, qui passe de l'individuel au social et au culturel, "des fantasmes du névrosé individuel aux créations fantasmatiques des peuples, telles qu'elles s'offrent à nous dans les mythes, les légendes et les contes ${ }^{22}$ ». Il renoue avec ses ambitions de jeunesse: il n'éprouvait aucune prédilection pour l'activité de médecin, mais il était mû par le désir de connaître la condition humaine ${ }^{23}$. Pourtant, il évita toujours de s'approcher de la philosophie : «les larges concordances de la psychanalyse avec la philosophie de Schopenhauer — il n'a pas seulement soutenu la thèse du primat de l'affectivité et de l'importance de la sexualité, mais il a même eu connaissance du mécanisme du refoulement - ne peuvent se déduire de ma familiarité avec sa doctrine. J'ai lu Schopenhauer très tard dans ma vie. Quant à Nietzsche, l'autre philosophe dont les pressentiments et les aperçus

17. L'avenir d'une illusion (1927), p. 20.

18. Mö̈se, p. 185.

19. Idem, p. 132.

20. "Quel sacrilège on a commis à l'encontre de la grandiose diversité de la vie humaine en ne voulant reconnaître que des mobiles tirés des besoins matériels » (Moïse, p. 128).

21. Sigmund Freud présenté par lui-même, pp. 17, 27.

22. Idem, p. 117.

23. Idem, p. 15. 
coïncident souvent de la manière la plus étonnante avec les résultats laborieux de la psychanalyse, je l'ai longtemps évité précisément pour cette raison; la priorité dans la découverte m'importait moins que de rester sans prévention ${ }^{24}{ }_{\text {». }}$ Très tôt, il se plongea dans l'histoire biblique, c'est-à-dire dans des récits épiques. C'est l'exposé de l'essai de Gœthe sur La nature par le Pr. Carl Brühl, qui décida de son inscription en médecine. Il se tourna vers les maladies nerveuses, les névroses, l'hystérie, la neuropathologie, bref, l'étude du cerveau, siège de la raison, de la volonté et des passions. Avec l'interprétation des rêves, la psychanalyse franchit les limites d'une spécialité médicale, trouvant des applications dans de multiples domaines : art, littérature, préhistoire ${ }^{25}$, mythologie, histoire des religions, ethnologie, pédagogie..., si bien qu' «il n'est plus possible de réserver l'exercice de la psychanalyse aux médecins ${ }^{26}{ }^{2}$.

" Je tiens personnellement en plus haute estime mes contributions à la psychologie de la religion ${ }^{27}$. » Comme Durkheim, Freud était très intéressé par le totémisme: "premier système d'organisation des tribus primitives, dans lequel les débuts d'un ordre social s'allient à une religion rudimentaire et au règne implacable d'un petit nombre de tabous ${ }^{28}{ }^{\prime}$. Deux en particulier - ne pas tuer l'animal totémique, ne nouer aucun commerce sexuel avec les femmes du même clan totémique - coïncident avec le complexe d'CEdipe : écarter le père et prendre sa mère pour femme. Il ne manquait plus grand-chose pour reconnaître dans le meurtre du père le noyau du totémisme et le point de départ de la constitution de la religion. Ce fragment manquant fut fourni par l'ouvrage de Robertson Smith: The Religion of the Semites. Il exposait que le repas totémique était l'élément essentiel de la religion totémique : une fois par an, l'animal totémique, qui était sacré le reste du temps, était tué solennellement avec la participation de tous les membres de la tribu; on le dévorait, puis on en faisait le deuil, suivi d'une grande fête. Processus similaire à celui du retour à la paix après la guerre.

Si j'associais tout cela à la conjecture darwinienne selon laquelle les hommes vivaient à l'origine en hordes dont chacune se trouvait sous la domination d'un mâle fort, violent et jaloux, je pouvais constituer à partir de toutes ces composantes une hypothèse, ou pour mieux dire : une vision, qui s'ordonnait de la manière suivante: le père de la horde primitive avait accaparé pour lui toutes les femmes, tué ou chassé tous les fils dangereux en tant que rivaux. Mais, un jour, ses fils s'unirent et, ensemble, le tuèrent et le dévorèrent, lui qui avait été leur ennemi, mais aussi leur idéal. Une fois l'acte accompli, ils furent incapables de prendre possession de son héritage, étant donné qu'ils se faisaient obstacle les uns aux autres. Sous l'effet de l'insuccès et du repentir, ils apprirent à s'accorder, ils se lièrent en un clan de frères par les réglements du totémisme, qui

24. Idem, p. 100.

25. Cf. "Sur la prise de possession du feu" (1932), in Résultats, idées, problèmes II, pp. 191-196.

26. Sigmund Freud présenté par lui-même, pp. 106, 118.

27. Idem, p. 112.

28. Idem, p. 113. 
devaient exclure le renouvellement d'une telle action, et ils renoncèrent globalement à la possession des femmes qui avaient été l'enjeu du meurtre du père. Ils étaient désormais renvoyés à des femmes étrangères; c'était là l'origine de l'exogamie, étroitement liée au totémisme. Le repas totémique était la cérémonie commémorative de l'acte monstrueux dont était dérivée la conscience coupable de l'humanité, par laquelle s'instaurèrent de manière concomitante organisation sociale, religion et limitation morale.

[Ainsi] « en se garantissant réciproquement la vie, les frères s'engagent à ne jamais se traiter les uns les autres comme ils ont tous traité le père. À l'interdiction de tuer le totem, s'ajoute l'interdiction du fratricide. Il se passera encore beaucoup de temps avant que cette interdiction, dépassant les limites du clan, devienne ce bref et clair commandement: tu ne tueras point ${ }^{29}$.

Freud en tirait la conclusion suivante: «La société repose sur un crime commis en commun; la religion, sur le sentiment de culpabilité et le repentir ; la morale, sur le besoin d'expiation engendré par le sentiment de culpabilité $^{30}$. » Mais ce sentiment, ainsi que celui de révolte du fils, ne disparaissent jamais complètement.

À l'origine des sociétés, il y a donc, sinon la guerre, en tout cas le meurtre, une violence collective meurtrière intraspécifique, ayant laissé sa trace mnésique $^{31}$. Voilà la thèse fondamentale de Freud, exposée dans Totem et tabou ${ }^{32}$, répétée dans des ouvrages ultérieurs, notamment Moïs $e^{33}$. Le sentiment de culpabilité, qui s'est condensé dans l'hypothèse chrétienne du péché originel, est l'expression d'un crime de sang dont s'est chargée l'humanité.

J'ai voulu deviner la nature de cette faute ancienne, et je pense que la doctrine chrétienne nous permet encore de la trouver par déduction. Si le fils de Dieu a été forcé de sacrifier sa vie pour délivrer l'humanité du péché originel, il faut selon la loi du talion - rendre la pareille - que ce péché ait consisté en un meurtre. Et si le péché originel fut une faute commise envers le Père-Dieu, il faut que le plus ancien crime de l'humanité ait été un parricide, le meurtre du père originaire de la horde humaine primitive, père dont l'image mnésique a été plus tard transfigurée en divinité ${ }^{34}$.

Utilisant la psychanalyse pour explorer les origines de la religion et de la morale, Freud place leur constitution sur le terrain du complexe paternel : le père originel devint le modèle de Dieu. L'auteur se tourne vers les peuples primitifs. Il y rencontre trois types de prescriptions : les tabous se rattachant aux ennemis, aux chefs, aux morts. Le meurtre d'un homme ne peut y être

29. Idem, pp. 114-115.

30. Totem et tabou, p. 205.

31. "Par le péché originel, la mort (violente) était entrée dans le monde » (Moïse, p. 178).

32. pp. 197-217.

33. pp. 235 sq.

34. "Considérations actuelles sur la guerre et la mort », pp. 30-31. 
accompli sans l'observance de certaines règles. Celles-ci se rangent en quatre groupes, selon qu'elles exigent la réconciliation avec l'ennemi, des pratiques cérémoniales, des actes d'expiation ou des restrictions. Dans le rapport à l'ennemi se manifestent d'autres sentiments que l'hostilité : le repentir, l'hommage, la reconnaissance de l'interdiction de l'homicide et du châtiment en cas de violation de l'interdit. "La tentation de tuer est plus forte en nous que nous ne le croyons $^{35}$. » Le tabou de l'homicide, comme le commandement de ne point tuer, loin d'être superflus, s'expliquent et se justifient précisément par l'attitude ambivalente à l'égard de l'impulsion au meurtre. Pour réprimer la tentation, il est proclamé et il est su que la transgression sera punie ${ }^{36}$. La loi ne défend que ce que les hommes sont capables de faire, en l'occurrence le meurtre : ce que la nature elle-même défend n'a pas besoin d'être défendu par la loi. Parmi les pulsions dont l'expression n'est pas totalement interdite, figure l'homicide judiciaire ou militaire. Fondamentalement, la psychologie freudienne de la guerre associe la religion ou la morale et la guerre, car elle se concentre sur l'homicide, sur la transgression ou la suspension de l'interdit de l'homicide, sur le sacrifice et la croyance en une vie métaphysique après la mort physique. Dans la guerre est levée l'interdiction de tuer autrui. Comment expliquer cette suspension et l'acceptation de cette suspension? Parce que l'homme a besoin d'une libération temporaire des renoncements que la civilisation impose à ses instincts. La guerre est comme la fête : elle est "un excès permis, voire ordonné, une violation solennelle d'un interdit ${ }^{37} »$.

Si la guerre a pour noyau le combat collectif, c'est-à-dire l'intention de tuer et l'acceptation du risque d'être tué ${ }^{38}$, elle dépend de l'agressivité ${ }^{39}$ et de l'esprit de sacrifice. Si la capacité de tuer et la capacité de se dévouer expliquent la propension à la guerre, la réflexion sur la guerre commence par l'interrogation suivante: d'où viennent l'agressivité et l'esprit de sacrifice? De la nature ou de la culture ? De l'inné ou de l'acquis ? L'origine de la violence estelle biologique ou sociologique ? La première difficulté est d'intégrer l'apport des sciences naturelles dans la recherche en sciences sociales, alors que les deux univers sont cloisonnés. La division entre sciences de l'homme et sciences de la nature explique l'écart entre l'étude du comportement humain et celle du

35. Totem et tabou, p. 103.

36. «Un nombre infini d'hommes de la culture, qui reculeraient d'effroi devant le meurtre, ne se refusent pas la satisfaction de leur désir s'ils peuvent le faire en restant impunis » (L'avenir d'une illusion, p. 12).

37. Totem et tabou, p. 198. Cf. aussi Gaston Bouthoul: Traité de polémologie, Paris, Payot, 1991, pp. 330-340.

38. Il y a guerre lorsqu'il y a réciprocité dans l'action violente appelée combat: la guerre ne commence pas lorsque certains individus en tuent d'autres, mais lorsqu'ils sont eux-mêmes prêts à risquer leur vie. Aussi est-elle l'activité la plus dangereuse: d'où la fascination qu'elle exerce. Cf. Martin van Creveld: La transformation de la guerre, Monaco, Rocher, 1991.

39. La lutte armée d'une collectivité contre une autre, de la même espèce, est la forme guerrière de l'agressivité. 
comportement animal, entre l'étude du comportement appris et celle du comportement inné. Cet écart s'explique aussi par des postulats religieux, moraux ou idéologiques, selon lesquels l'homo n'est ni un animal ni le produit d'une évolution génétique. Freud, décloisonnant les disciplines, s'intéresse à l'origine psychologique de la violence, cependant qu'il souligne la continuité des phénomènes normaux et des phénomènes pathologiques. De nombreuses espèces ont une inhibition biologique à tuer d'autres individus appartenant à la même espèce. On ne la trouve pas chez l'homme: l'homicide est interdit à l'intérieur du groupe, il ne l'est pas à l'extérieur, du moins en temps de guerre, la hiérarchie politique empêchant les membres du groupe de se battre entre eux mais pas avec des individus d'autres groupes. Au cours de l'évolution de l'humanité, les mécanismes biologiques inhibiteurs de la violence intra-spécifique ont été remplacés par des mécanismes sociologiques, exprimés par les langages normatifs que sont la religion, la morale, le droit: "tu ne nuiras pas à autrui ", du moins en temps de paix et à l'intérieur du groupe. Il y a un rapport entre l'efficacité des armes d'une espèce et les inhibitions qui empêchent l'emploi intra-spécifique de ces armes : à l'invention de l'arme, chez l'humain, a répondu l'invention de la responsabilité morale. L'agressivité est définie comme la propension innée à l'exercice de la violence contre un individu de la même espèce dans le dessein de lui nuire : ce que l'éthologie appelle "l'instinct de combat », la psychanalyse, la «pulsion de mort $»^{40}$.

Après l'introduction de la pulsion de mort dans la théorie psychanalytique, Malaise dans la culture (1930) s'intéresse avant tout à l'agressivité dirigée vers l'extérieur. Ce déplacement de l'intériorité aux relations entre les hommes conduit Freud hors du champ propre de la psychanalyse vers la sociologie. L'ouvrage est une étude psychologique sur la genèse de la religion. Ses huit chapitres pourraient s'intituler comme suit: le narcissisme ou «sentiment océanique du moi », origine du sentiment religieux; la recherche du bonheur contre la souffrance que peuvent causer le corps, la nature et autrui ; la culture, instrument de l'homme vis-à-vis de la nature et d'autrui ; la difficulté du renoncement pulsionnel et l'hétérosexualité monogamique; la difficulté du renoncement pulsionnel et l'amour du prochain; la pulsion de vie (l'amour) et la pulsion de mort (l'agressivité) ; l'origine du sentiment de culpabilité et de la conscience morale; le sentiment de culpabilité, problème $\mathrm{n}^{\circ} 1 \mathrm{du}$ développement de la culture. Des trois sources de notre souffrance: la surpuissance de la nature, la caducité de notre corps, la déficience des dispositifs qui règlent les relations des hommes entre eux, c'est la dernière - la souffrance sociale - qui nous révolte le plus : on admet, même de plus en plus difficilement, que nous ne dominerons jamais complètement la nature et que notre corps est mortel. Par contre, on voudrait que les rapports interindividuels

40. Cf. Konrad Lorenz: L'agression, Paris, Flammarion, 2000 (1969); Revue internationale des sciences sociales: Comprendre l'agressivité, 1971; Viviana Pâques : «La guerre dans les sociétés traditionnelles ", in Jean Poirier (dir.) : Histoire des mours, Paris, Gallimard, 1991, vol. 1, III, "L'homme et la guerre », pp. 347-381. 
dans la famille, la société, l'État, les relations internationales, deviennent harmonieux. La question décisive est donc de savoir si le développement de la culture permettra de se rendre maitre de la pulsion naturelle d'agression. Tel est le combat de titan entre Eros et Thanatos.

Grâce à sa technologie, l'homme moderne est «devenu une sorte de dieu prothétique $^{41}$ ». Mais l'homme moderne "ne se sent pas heureux dans sa ressemblance avec Dieu ${ }^{42}$ ». C'est que la vie moderne s'accompagne d'un important renoncement à la vie pulsionnelle: «La culture est édifiée sur du renoncement pulsionnel, elle présuppose la non-satisfaction de puissantes pulsions $^{43}$." Le report de satisfaction est la condition du progrès. Si la culture impose d'aussi grands sacrifices, à la sexualité comme "au penchant de l'homme à l'agression ${ }^{44}$ ", on comprend pourquoi il est difficile à l'homme de s'y trouver heureux. "L'homme de la culture a fait l'échange d'une part de bonheur contre une part de sécuritét ${ }^{45}$. " Si la civilisation doit s'édifier sur la contrainte et le renoncement, réussira-t-on, et jusqu'à quel point, à diminuer le fardeau qu'est le sacrifice des pulsions? "Les masses n'aiment pas le renoncement pulsionnel ${ }^{46}$. » L'agressivité, «ce trait indestructible de la nature humaine ${ }^{47}$ ", menace constamment la société civilisée et doit être inhibée. C'est la pulsion de vie qui a pour tâche de rendre inoffensive la pulsion de mort: la libération sexuelle doit satisfaire les pulsions et détourner l'agressivité. Lorsqu'une part des puissances inhibitrices à l'œuvre dans le monde extérieur est intériorisée, il se forme dans le moi une instance qui se pose en face du reste pour observer et interdire: la conscience ou le surmoi. Le surmoi est le successeur des parents. Il devrait être le substitut du dieu éthique personnel. Le progrès de la vie de l'esprit consiste en ce que les processus intellectuels supérieurs s'imposent aux perceptions sensorielles primaires. La vie de l'esprit peut cependant être dominée par un phénomène émotionnel: celui de la foi. Mais :

la religion qui a commencé par l'interdiction de se faire une image de Dieu se développe toujours dans le sens d'une religion des renoncements aux pulsions. Dieu est détaché de la sexualité pour être élevé à un idéal de perfection éthique. Or éthique signifie limitation des pulsions. Dieu n'exige rien d'autre de son peuple qu'il s'abstienne de toutes les satisfactions pulsionnelles ${ }^{48}$.

41. Malaise dans la culture, pp. 34-35.

42. Idem, p. 35.

43. Idem, p. 41.

44. Idem, p. 57.

45. Idem, p. 57.

46. Idem, p. 55.

47. Idem, p. 56.

48. Idem, p. 61. 
Même la doctrine de la guerre juste ou sainte ${ }^{49}$ exige une «intention droite ", le respect des règles du combat, la ritualisation et «l'impersonnalité active ": on ne combat pas pour soi, mais pour une Idée, une cause collective ou transcendante.

La religion comme la guerre ayant pour centre psychologique l'idée du sacrifice, la psychanalyse de la religion concerne la psychanalyse de la guerre. L'avenir d'une illusion (1927) ou l'analyse critique de la psychogénèse de la religion, est construit en dix chapitres qui pourraient s'intituler comme suit : la culture, la contrainte et le renoncement pulsionnel; l'intériorisation de la contrainte et du renoncement; la religion, compensation et consolation de la finitude humaine; le totémisme, origine de la religion, en tant que protection maternelle (la mère protège l'enfant), paternelle (le père protège la famille) et divine (dieu protège la communauté) ${ }^{50}$; l'irrationalité des représentations religieuses; les religions comme illusions; le danger culturel du déni de $D_{i e u^{51}}$; la religion, névrose œdipienne; de la possibilité d'en finir avec l'illusion religieuse; de la possibilité de surmonter la religion par la science. Freud plaide pour un fondement rationnel des prescriptions de la culture, permettant de ne plus imputer à Dieu la volonté des hommes. Mais il peut être dangereux que les masses ne croient plus à Dieu. D'autant que le commandement chrétien d'aimer son prochain constitue la plus forte barrière morale à l'agressivité. D'un autre côté, si la religion est l'une des sources de la guerre ou de sa justification, la déconstruction de l'illusion religieuse va dans le sens de la paix. Si Dieu n'est qu'un "concept vide $e^{52}$ ", comment et pourquoi se battre en son nom?

\section{La société moderne et la mort violente}

Les Considérations sur la guerre et la mort comprennent deux parties, rédigées en mars et avril 1915 : l'une étant «La désillusion causée par la

49. Cf. Jean Flori: Guerre sainte, jihad, croisade. Violence et religion dans le christianisme et l'islam, Paris, Points Seuil, 2002.

50. Après l'animal totémique, viennent la Grande Mère, ses filles et fils, puis apparaissent les dieux mâles et leurs épouses, enfin le Père, son Fils. Telle est l'évolution du sentiment religieux vue d'Europe.

51. « De la part des gens cultivés, la culture n'a pas grand-chose à redouter. Remplacer les motifs religieux d'un comportement culturel par d'autres motifs, profanes, cela se ferait chez eux sans bruit. Il en va autrement pour la grande masses des gens incultes qui ont toute raison d'être des ennemis de la culture. Aussi longtemps qu'ils n'apprennent pas qu'on ne croit plus en Dieu, cela va bien. Mais ils l'apprendront immanquablement. Et il sont prêts à adopter les résultats de la pensée scientifique, sans que soit instaurée en eux la modification que la pensée scientifique entraîne chez l'homme. Si on ne doit pas tuer son prochain pour la seule raison que Dieu a interdit cet acte et le sanctionnera dans cette vie ou dans l'autre, mais si l'on apprend qu'il n'y a pas de Dieu et qu'il n'y a pas lieu de craindre sa punition, alors on n'aura sûrement aucun scrupule à abattre ce prochain et l'on ne pourra en être empêché que par un pouvoir terrestre. Ainsi donc, ou bien tenir dans la plus stricte sujétion ces masses dangereuses, ou bien réviser fondamentalement la relation entre culture et religion" (L'avenir d'une illusion, p. 40).

52. Idem, p. XI. 
guerre ", l'autre "Notre relation à la mort ». Freud y révèle sa compréhension psychologique de la guerre industrielle de masse prolongée. L'entrée dans le conflit armé avait surpris Freud, qui n'accordait que peu d'attention à la situation internationale de l'époque. Lui aussi s'était trouvé plongé au début dans un enthousiasme enivrant: "toute ma libido va à l'Autriche-Hongrie ${ }^{53}$ ". Contrairement à ce qu'avancent la plupart des commentateurs, les deux parties susmentionnées ne développent pas une critique des « idées de 1914 ». Elles ne font aucune allusion au nationalisme. Elles invitent à une introspection sociologique sur la morale et la mort violente. Les Considérations peuvent être complétées par l'étude de 1921 sur la Psychologie des foules.

1) La guerre a provoqué une "désillusion» et un "changement d'attitude à l'égard de la mort ${ }^{54} »$. Cette transformation n'affecte pas que les soldats du front mais les civils de l'arrière ("l'individu qui n'est pas devenu lui-même un combattant et, de ce fait, une infime particule de la gigantesque machine de guerre $)^{55}$. La "misère psychique de ceux de l'arrière ${ }^{56}$ » s'explique par l'évanouissement d'un espoir. On disait que les guerres ne pourraient cesser tant que les peuples auraient des conditions d'existence très différentes, tant que l'appréciation des valeurs relatives à la vie des individus serait aussi divergente, tant que les haines qui les séparent représenteraient de si grandes forces pulsionnelles. "Aussi était-on préparé à ce que des guerres entre les peuples primitifs et civilisés, entre les races de couleurs différentes, retiennent pendant longtemps encore l'attention de l'humanité. » Mais :

on osait espérer quelque chose d'autre. Des grandes nations de race blanche régnant sur le monde, auxquelles incombe la direction du genre humain, dont l'œuvre comprend aussi bien les progrès techniques dans la domination de la nature que les valeurs artistiques et scientifiques de civilisation - de ces peuples-là, on avait attendu qu'ils fussent capables de résoudre par d'autres voies les conflits d'intérêts. Au sein de chacune de ces nations avaient été établies, pour l'individu, des normes morales élevées (qui) exigeaient un large renoncement à la satisfaction pulsionnelle ${ }^{57}$.

Il lui était fondamentalement interdit de nuire à autrui. «L'État civilisé considérait ces normes morales comme les assises de son existence. On pouvait donc supposer qu'il les respecterait luimême $^{58}$. " Dans une telle communauté de civilisation, développée et

53. Cité par H. Glaser, p. 178.

54. "Considérations actuelles sur la guerre et la mort », p. 10.

55. Idem, p. 9.

56. Idem, p. 10.

57. Idem, pp. 10-11.

58 Idem, p.11. 
évoluée, comment se figurait-on la guerre, si tant est qu'on en arrivait là ? "Comme une occasion de montrer les progrès accomplis par les hommes dans le sentiment de leurs intérêts communs ${ }^{59}$. " Le tournant du siècle n'avait-il pas vu la codification du jus in bello ${ }^{60}$ ?

Comme une passe d'armes chevaleresque qui se bornerait à constater la supériorité d'une des parties, en évitant le plus possible les souffrances qui ne sauraient contribuer en rien à la décision, en épargnant le blessé, le médecin, la partie non belligérante de la population, les femmes et les enfants, les institutions internationales dans lesquelles s'était incarnée la communauté de civilisation du temps de paix ${ }^{61}$.

Une telle guerre n'aurait pas manqué d'épreuves, «mais elle n'aurait pas interrompu le développement de rapports moraux entre ces grands individus de l'humanité que sont les peuples ${ }^{62}$.» Et voilà qu'éclata la guerre "à laquelle nous ne voulions pas croire " : aussi cruelle et plus meurtrière que celles du passé du fait du perfectionnement des armes, rejetant les limitations et les distinctions du droit des gens, menaçant de laisser derrière elle une animosité qui empêcherait les peuples en conflit de renouer leurs liens. Les "peuples civilisés» se retournent, pleins de haine, les uns contre les autres. L'État en guerre se permet toutes les violences, exige de ses citoyens le maximum d'obéissance et de sacrifices, s'affranchit de tous les traités. Le relâchement des rapports moraux entre les États, qui se comportaient à l'intérieur comme les gardiens de la morale, ne peut qu'avoir des répercussions sur la moralité des individus. La désillusion qu'a suscitée la guerre porte sur l'État, l'individu et la civilisation. Elle signifie la fin de la croyance au progrès moral de l'humanité et, dira George L. Mosse, l'avènement de la «brutalisation » des sociétés européennes ${ }^{63}$.

Comment un individu parvient-il à un degré supérieur de moralité ? Si l'on ne croit pas en la bonté naturelle de l'homme ( « ne nous y attardons pas davantage $")^{64}$, on pensera que c'est l'éducation qui compte. Comment expliquer alors que, chez l'homme ainsi éduqué, le mal réapparaisse dans toute sa virulence? "En vérité, la recherche psychanalytique montre que l'essence de l'homme consiste en motions

59. Idem, pp. 12-13.

60. Cf. James Brown Scott: Les Conférences de la Paix de La Haye de 1899 et 1907, Paris, Pedone, 1927.

61. "Considérations actuelles sur la guerre et la mort ", p. 13.

62. Idem, p. 13.

63. Cf. De la Grande Guerre au totalitarisme. La brutalisation des sociétés européennes, Paris, Hachette, 1999 (1990), préf. S. Audoin-Rouzeau.

64. "Considérations actuelles sur la guerre et la mort », p. 16. 
pulsionnelles qui tendent à la satisfaction de certains besoins originels ${ }^{65}$." Ces pulsions ne sont en soi ni bonnes ni mauvaises : nous ne les classons telles qu'en fonction des besoins et des exigences de la société ( « le caractère d'un homme ne peut être classé que très insuffisamment à l'aide des mots 'bon' et 'mauvais' $)^{66}$. L'influence exercée sur les pulsions égoïstes par le besoin d'amour les transforment en pulsions altruistes. "On apprend à voir dans le fait d'être aimé un avantage qui permet de renoncer à tous les autres ${ }^{67}$. " L'éducation représente la contrainte extérieure, sous la forme de la récompense et de la punition. "En donnant à la capacité impartie à un homme de remanier ces pulsions égoïstes sous l'influence de l'érotisme le nom d'aptitude à la civilisation, nous pouvons dire que celle-ci se compose de deux parties, l'une innée et l'autre acquise ${ }^{68}$." La société qui exige que l'on agisse bien, sans se soucier du fondement pulsionnel de l'action, a ainsi fait qu'un grand nombre d'individus obéissent à la civilisation sans suivre en cela leur nature. Ils se trouvent contraints à une constante répression pulsionnelle, et la tension qui en résulte se traduit par des phénomènes de réaction ou de compensation. "Celui qui est obligé de réagir constamment dans le sens de prescriptions qui ne sont pas l'expression de ses penchants pulsionnels, vit, psychologiquement parlant, au-dessus de ses moyens et mérite objectivement d'être qualifié d'hypocrite ${ }^{69}$. " Il y a ainsi plus d' " hypocrites de la civilisation que d'hommes authentiquement civilisés ${ }^{70}{ }$. C'est pourquoi la désillusion provoquée par la guerre n'est finalement pas justifiée: elle reposait sur une illusion, celle de l'élévation morale de l'humanité européenne. "Nos concitoyens du monde ne sont pas tombés aussi bas que nous le redoutions, parce qu'ils ne s'étaient pas élevés aussi haut que nous l'avions pensé ${ }^{71}$. " La guerre n'a fait que révéler l'« extraordinaire plasticité des développements psychiques ${ }^{72}$ »: leur capacité à progresser comme à régresser. «Le remaniement pulsionnel sur lequel repose notre aptitude à la civilisation, peut être ramené en arrière; les influences exercées par la guerre sont au nombre des forces capables de produire un tel retour en arrière ${ }^{73}$." Voilà la formule clé: "les états primitifs peuvent toujours être réinstaurés ${ }^{74}{ }^{»}$. Freud démolit ce qu'on appelle aujourd'hui la théorie de

65. Idem, p. 16.

66. Idem, p. 17.

67. Idem, p. 18.

68. Idem, p. 18.

69. Idem, p. 20.

70. Idem, p. 21.

71. Idem, p. 21.

72. Idem, p. 22.

73. Idem, p. 22.

74. Idem, p. 22. 
la paix par la démocratie ou le développement: des peuples hautement évolués sont tout à fait capables de, voire disposés à, se lancer dans la guerre. "Nous avions espéré que l'impressionnante communauté d'intérêts née de l'échange et de la production » garantirait la paix; "mais il semble que les peuples obéissent, pour l'instant, beaucoup plus à leurs passions; ils mettent en avant leurs intérêts pour trouver des raisons à la satisfaction de leurs passions ${ }^{75}$. » L'intelligence, quelle petite chose à la surface de nous-mêmes : Freud en était bien persuadé $^{76}$ ! Comme dira Bouthoul, la décision des gouvernants de recourir aux armes doit rencontrer l'assentiment des gouvernés. Or cette acceptation, comme cette décision, dérivent d'impulsions passionnelles: la guerre est un rite d'exaltation collective $\mathrm{e}^{77}$.

2) La guerre a évidemment transformé le rapport à la mort. S'appuyant sur le matériel de Totem et tabou, Freud observe que dans la société européenne d'avant-guerre, hautement civilisée, ce rapport «manquait de franchise ${ }^{78}$ ». Tout le monde admet que la mort est naturelle, donc inévitable. Cependant, chacun tend à la mettre de côté, à l'oublier, à l'éliminer de la vie.

C'est que notre propre mort ne nous est pas représentable et aussi souvent que nous tentons de nous la représenter, nous pouvons remarquer (que) nous continuons à être là en tant que spectateur. C'est pourquoi dans l'école psychanalytique, on a pu oser cette déclaration: personne, au fond, ne croit à sa propre mort ou : dans l'inconscient, chacun de nous est persuadé de son immortalité 79 .

Les adultes civilisés ne parlent pas de la mort d'autrui si autrui est leur interlocuteur ou peut l'entendre. Seuls les enfants passent outre cette limitation et peuvent dire à une personne aimée qu'elle va mourir. L'homme est ainsi l'animal qui sait qu'il va mourir et qui

75. Idem, p. 25.

76. «Il est chez nos concitoyens du monde un autre symptôme qui ne nous a peut-être pas moins surpris que l'abaissement si douloureusement ressenti de leur grandeur morale. Je veux parler du manque de jugement, qui se manifeste chez les meilleures têtes, de leur obstination, de leur inaccessibilité aux arguments les plus convaincants. Cela donne assurément un triste tableau et je veux affirmer bien haut que, nullement aveuglé par le parti pris, je ne trouve pas ces errements intellectuels seulement dans l'un des deux camps. Mais ce phénomène est encore plus aisé à expliquer et beaucoup moins préoccupant. Nous avons appris depuis longtemps que nous avons tort de considérer notre intelligence comme une force autonome et de ne pas voir combien elle dépend de la vie affective. Notre intellect ne pourrait travailler de façon sûre que soustrait à l'action de fortes motions affectives; dans le cas contraire, il se comporterait simplement comme un instrument entre les mains d'une volonté. Les arguments logiques seraient sans pouvoir contre les intérêts affectifs, et c'est pourquoi la lutte à coup de raisons est, dans le monde des intérêts, si stérile» (idem, pp. 23-24).

77. G. Bouthoul, op. cit., p. 332.

78. "Considérations actuelles sur la guerre et la mort », p. 26.

79. Idem, p. 26. 
refoule cette réalité. On ne met l'accent que sur « la cause occasionnelle de la mort ${ }^{80} »$ : accident, maladie, vieillesse, guerre. À l'égard du mort, le comportement des vivants est particulier : toute critique est suspendue et l'oraison funèbre ne célèbre que ses mérites. Cette attitude conventionnelle, liée à la civilisation, est complétée par notre chagrin quand la mort a frappé l'un de nos proches. Cette relation à la mort, basée sur le refoulement, nous rend impossible d'admettre que l'on puisse sacrifier sa vie. "La vie s'appauvrit dès l'instant où il n'est plus possible de risquer la mise suprême, c'est-à-dire la vie ellemême. " Elle devient " aussi vide qu'un flirt américain dans lequel il est établi d'emblée qu'il ne se passera rien, à la différence d'une relation amoureuse continentale dont les graves conséquences doivent toujours être présentes à l'esprit des deux partenaires ". L'intensité de notre deuil fait que "nous sommes peu enclins à rechercher le danger pour nous et pour les nôtres. La pensée qui nous paralyse, c'est de savoir qui, en cas de malheur, remplacera pour la mère le fils, pour l'épouse l'époux, pour les enfants le père. La tendance à exclure la mort des comptes de la vie a pour conséquence bien d'autres renoncements $^{81}$.» C'est pourquoi on cherche dans l'univers de la fiction, littérature, théâtre, cinéma, "un substitut à ce que la vie nous fait perdre. C'est là que nous trouvons encore des hommes qui savent mourir ${ }^{82}$. " La guerre balaie la manière conventionnelle de traiter la mort : celle-ci ne se laisse plus refouler; "on est forcé de croire en elle $^{83}{ }^{\prime \prime}$. Les hommes meurent réellement, en masse, parce qu'ils sont tués $^{84}$. "La vie est redevenue intéressante, elle a retrouvé tout son contenu $^{85}$. » Deux groupes apparaissent clairement : «ceux qui font le sacrifice de leur vie ${ }^{86}$, ceux qui sont restés à la maison et n'ont qu'à attendre de perdre un être $\operatorname{cher}^{87}{ }^{8}$. Voilà le vrai clivage du temps de guerre selon Freud: non pas ami/ennemi, mais combattants/non combattants, ceux qui sont en situation de tuer et d'être tués, ceux qui ne le sont pas. Sur la psychologie des combattants, Freud sait « trop peu de choses » : « il faut nous en tenir au second groupe auquel nousmêmes appartenons ${ }^{88}$.»

80. Idem, p. 27.

81. Idem, p. 28.

82. Idem, pp. 28-29.

83. Idem, p. 29.

84. "Mépris de la mort », tel était le mot clé de l'époque, observe H. Glaser : ceux qui détenaient le pouvoir de commandement étaient prêts à sacrifier leurs prochains, ceux qui étaient sacrifiés acceptaient de mourir (p. 205).

85. "Considérations actuelles sur la guerre et la mort », p. 29.

86. Les fils de Freud.

87. Freud lui-même. Quelle est la psychologie du père dont les fils partent à la guerre alors que lui-même n'y part pas ? Fierté, jalousie, culpabilité ?

88. «Considérations actuelles sur la guerre et la mort », p. 29. 
Pour surmonter le désarroi face à la mort violente, la recherche psychologique, selon notre auteur, devrait porter sur l'homme des origines d'une part, sur l'inconscient d'autre part. Comment l'homme des origines s'est-il comporté face à la mort ? Il a reconnu la mort en même temps qu'il l'a niée. Cette contradiction s'explique par le fait qu'il avait sur la mort de l'autre une position différente de celle qu'il avait sur sa propre mort, s'accommodant fort bien de la première, mais évidemment pas de la seconde. L'homme des origines est un animal dangereux et méchant, n'ayant pas d'inhibition biologique au meurtre intra-spécifique et le pratiquant. "Aussi l'histoire des origines de l'humanité est-elle pleine de meurtres. Aujourd'hui encore, ce que nos enfants apprennent à l'école sous le nom d'Histoire est pour l'essentiel une suite de meurtres de peuples à peuples ${ }^{89}$. » Telle est bien la définition de la guerre : lutte armée et sanglante entre groupements organisés (G. Bouthoul). Lorsque l'homme des origines voyait mourir un de ses proches, il lui fallait dans la douleur faire l'expérience que lui-même était mortel, même si tout son être se révoltait contre une telle reconnaissance. Chacune de ses personnes chères était à la fois une part de lui-même et une personne étrangère à lui-même. C'est l'énigme de la mort qui a contraint l'homme à la spéculation. Il s'engagea dans un compromis : il accepta que la mort fût aussi pour lui, mais contesta sa signification en tant qu'anéantissement. L'angoisse devint le fondement de la croyance en d'autres formes d'existence et en la survie après la mort apparente. Freud cite le mythe d'Achille ${ }^{90}$ «Auprès du cadavre de la personne aimée prirent naissance non seulement la doctrine de l'âme, la croyance en l'immortalité, et l'une des puissantes racines de la conscience de culpabilité chez l'homme,

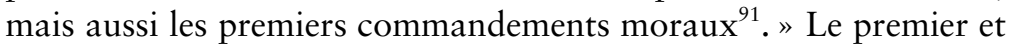
le plus significatif des interdits fut: tu ne tueras point, qui s'étendit des proches aux étrangers. Un interdit si puissant, universel et réitéré, ne peut se dresser que contre une impulsion d'égale puissance : ce qu'on ne désire pas, on n'a pas besoin de l'interdire. "Précisément, le caractère insistant du commandement: tu ne tueras point, nous donne la certitude que nous descendons d'une lignée infiniment longue de meurtriers qui avaient dans le sang le désir de tuer, comme peut-être nous-mêmes encore ${ }^{92}$. » Les aspirations morales de l'humanité sont ainsi un héritage de son histoire belliqueuse.

89. Idem, p. 30.

90. Cf. Eric Desmons: Mourir pour la patrie? Paris, PUF, 2001, «Le mythe de la belle mort d'Achille ", pp. 17-25.

91. "Considérations actuelles sur la guerre et la mort », p. 34.

92. Idem, p. 35. 
D'après Freud, l'homme des origines survit inchangé dans notre inconscient. Celui-ci ne croit pas à la mort personnelle. "Peut-être est-ce là le secret de l'héroïsme » : plutôt que l'acceptation du sacrifice au nom d'une cause collective ou transcendante, "l'héroïsme instinctif et impulsif brave les dangers avec l'assurance » de celui qui croit que rien ne peut lui arriver ${ }^{93}$. D'autre part, on trouve légitime la mort des ennemis. C'est ainsi que l'on passe outre à l'interdiction de tuer, cependant que l'on croie obscurément que la mort apparente ne marque pas une fin réelle. En résumé:

Tout autant que l'homme des temps originaires, notre inconscient est inaccessible à la représentation de notre propre mort, est plein de désirs meurtriers à l'égard de l'étranger, est ambivalent à l'égard de la personne aimée. Comme l'atti-tude conventionnelle à l'égard de la mort nous a éloignés de cet état originaire! Il est facile de dire de quelle façon la guerre intervient dans ce désaccord. Elle nous dépouille des couches récentes déposées par la civilisation et fait réapparaitre en nous l'homme des origines. Elle nous contraint de nouveau à être des héros qui ne peuvent croire à leur propre mort ; elle nous désigne les étrangers comme des ennemis dont on doit provoquer ou souhaiter la mort; elle nous conseille de ne pas nous arrêter à la mort des personnes aimées ${ }^{94}$.

D'autre part, la guerre ne se laisse pas éliminer. «Aussi longtemps que les peuples auront des conditions d'existence si différentes et que leur répulsion mutuelle sera si violente, il y aura nécessairement des guerres $^{95}$."

Que les conditions d'existence deviennent homogènes et que la répulsion s'évanouisse, sous l'effet de l'éducation ou des échanges par exemple, et la guerre disparaitrait-elle? Freud ne se demande pas comment mettre fin à la guerre, mais comment s'adapter à la guerre.

Ne devons-nous pas convenir qu'avec notre attitude de civilisé à l'égard de la mort nous avons, une fois encore, vécu psychologiquement au-dessus de nos moyens et ne devons-nous pas faire demi-tour et confesser la vérité ? $\mathrm{Ne}$ vaudrait-il pas mieux faire à la mort la place qui lui revient et laisser un peu plus se manifester notre attitude inconsciente à l'égard de la mort, que nous avons jusqu'à présent si soigneusement réprimée ? Cela ne semble pas être un progrès, plutôt une régression, mais cela présente l'avantage de mieux tenir compte de la vraisemblance et de nous rendre la vie de nouveau plus supportable. Supporter la vie reste bien le premier devoir de tous les vivants. L'illusion perd toute valeur quand elle nous en empêche ${ }^{96}$.

Si vis pacem, para bellum, disait le vieil adage: si vis vitam, para mortem, devrait-on dire.

93. Idem, p. 36.

94. Idem, p. 39.

95. Idem, pp. 39-40.

96. Idem, p. 40. 
3) Au noyau de la guerre, il y a l'homicide; au noyau de la révolution, il y a la foule. Freud en a brossé la psychologie. Il s'intéresse aux caractères des foules révolutionnaires. "L'individu se trouve, dans la foule, mis dans des conditions qui lui permettent de se débarrasser des refoulements de ses motions pulsionnelles inconscientes ${ }^{97}$. » C'est dans de telles conditions que, notamment, la conscience morale disparaît et que l'individu tombe dans une sorte d'état hypnotique où il n'est plus vraiment conscient de ses actes. Comme l'observait Gustave Le Bon, "par le fait seul qu'il fait partie d'une foule, l'homme descend plusieurs degrés sur l'échelle de la civilisation. Il a la spontanéité, la violence, les enthousiasmes et les héroïsmes des êtres primitifs ${ }^{98}$. " La foule est suggestible, elle a le sentiment de toutepuissance, elle ne connaît pas de doute. Elle "est aussi intolérante que pleine de foi dans l'autorité. Elle respecte la force. Ce qu'elle exige de ses héros, c'est de la brutalité. Elle veut être dominée ${ }^{99}$. " Dans le rassemblement en foule, "toutes les inhibitions individuelles tombent et tous les instincts destructeurs sont réveillés ${ }^{100}$.» Freud voit en l'Église et l'Armée - les deux institutions androcratiques de la religion et de la guerre - des « foules hautement organisées, durables et artificielles ${ }^{101}$ ». Elles sont " artificielles » parce qu'une certaine contrainte extérieure est mise en œuvre pour les préserver de la dissolution. «Dans l'Église prévaut, comme dans l'Armée, le même mirage qu'un chef suprême est là — dans l'Église catholique le Christ, dans l'armée le commandant en chef - qui aime tous les individus de la foule d'un égal amour ${ }^{102}$. » Telle est la structure libidinale sans laquelle les deux institutions s'effondreraient. Le "militarisme prussien» en a fait l'expérience en 1918, lorsque les «névroses de guerre » désagrégèrent l'armée allemande ${ }^{103}$. Le soldat prend pour idéal son supérieur, cependant qu'il s'identifie à ses semblables et qu'il fait découler de cette communauté les obligations propres à la camaraderie : loyauté, dévouement, sacrifice.

Dans Totem et tabou, rappelle Freud, j'ai adopté la supposition de Darwin selon laquelle la forme originaire de la société humaine serait celle

97. «Psychologie des foules et analyse du moi », p. 129.

98. Idem, p. 132.

99. Idem, p. 134.

100. Idem, p. 134.

101. Idem, p. 153.

102. Idem, p. 154.

103. Idem, p. 155. "Que l'essence d'une foule réside dans les liens libidinaux présents en elle ", on en trouve la preuve dans "le phénomène de la panique, qui s'étudie au mieux sur les foules militaires: plus aucun ordre du chef n'est écouté et chacun se préoccupe de lui-même sans se soucier des autres » (pp. 156-157). 
d'une horde soumise à la domination d'un mâle puissant. J'ai essayé d'exposer que les destins de cette horde ont laissé des traces dans l'histoire héréditaire de l'humanité, qui se rattache au meurtre du chef et à la transformation de la horde du père en une communauté de frères ${ }^{104}$.

La psychologie de la foule - disparition de la personnalité individuelle consciente, orientation des pensées et des sentiments dans des directions identiques, prédominance de l'affectivité et de l'inconscient, tendance à la réalisation immédiate de desseins qui surgissent — correspond à l'état de régression vers une activité psychique primitive, telle qu'on pourrait l'assigner à la horde originaire. De même que l'homme des origines s'est maintenu virtuellement en chaque individu, de même la horde originaire peut se reconstituer à partir de n'importe quel agrégat humain. L'homme redevient alors le membre d'une horde menée par un chef. Il en va tout particulièrement ainsi en temps de guerre. "Nous devons en conclure que la psychologie de la foule est la plus ancienne psychologie de l'homme ${ }^{105}$. " Il y eut deux sortes de psychologie au début : celle des individus en foule, celle du chef. "Au seuil de l'histoire de l'humanité était le surhomme que Nietzsche n'attendait que de l'avenir. Aujourd'hui encore, les individus en foule ont besoin de l'illusion d'être aimés par le meneur, mais le meneur, lui, n'a besoin d'aimer personne d'autre, il a le droit d'être de la nature des maîtres ${ }^{106}$. "C'est ainsi que le meneur de la foule est le père originaire et que le père originaire est l'idéal de la foule.

\section{«Pourquoi la guerre?»}

L'ouvrage se présente comme une réponse épistolaire de Freud à Einstein, qui lui demandait son avis de psychanalyste sur le phénomène, dans le cadre d'un échange de vue entre intellectuels de renom proposé par la Société des Nations pour servir la cause de la paix ${ }^{107}$. Le texte résume les recherches freudiennes

104. Idem, p. 189.

105. Idem, p. 191.

106. Idem, p. 191.

107. En 1931, le Comité des lettres et des arts de la SDN suscita un échange épistolaire entre "des personnalités marquantes du monde intellectuel pour discuter des questions qui concernent éminemment nos intérêts spirituels communs et servent la Société des Nations ». La Commission internationale pour la coopération intellectuelle s'adressa, pour concrétiser cette idée, à Albert Einstein, entre autres, en lui laissant la liberté de choisir son sujet et son correspondant. Il proposa Freud. Celui-ci accepta, bien qu'Einstein ne lui fût pas particulièrement proche. Il l'avait rencontré une fois, fin 1926, à Berlin: "Il est gai, sûr de lui et aimable, il est aussi au fait de la psychologie que moi de la physique, c'est pourquoi nous avons eu ensemble une agréable discussion. » Freud n'était pas enthousiasmé par cet échange épistolaire; il en parlait comme d'une discussion factice, ennuyeuse et stérile avec Einstein. Cette correspondance n'en fut pas moins publiée en mars 1933 à Paris en trois langues (allemand, français et anglais), mais sa diffusion fut interdite en Allemagne. 
dans ce domaine : l'énorme danger de la pulsion d'agression et la faiblesse concomitante de la raison humaine. Le principal problème de la civilisation est de savoir s'il y a un moyen de libérer les hommes de la fatalité de la guerre : c'est ainsi qu'Einstein commence sa lettre du 30 juillet 1932. Les décideurs politiques sont prêts à écouter les points de vue objectifs des hommes de science. Aussi espérait-il que Freud serait à même de proposer des mesures éducatives susceptibles d'écarter les obstacles psychologiques à une solution du problème. Pour Einstein, la difficulté est moins politique ${ }^{108}$ que psychologique. Comment faire pour que la minorité dirigeante se mette au service de la masse du peuple, qui n'a dans la guerre qu'à perdre et à souffrir ? Comment expliquer que la masse soit prête aux enthousiasmes et aux sacrifices guerriers? Y auraitil une possibilité de diriger le développement psychique des hommes de manière à les rendre plus résistants aux psychoses de la destruction? Par làmême se trouvent abordées les questions politiques fondamentales: le rapport du droit, du pouvoir, de la violence et de l'agressivité d'une part, les chances d'une éducation à la paix d'autre part. Si l'agression est "naturelle », elle n'est pas le «mal »; peut-elle être contenue, déviée ou limitée par la culture ? Il y a deux pulsions fondamentales en l'homme: celles qui veulent conserver et aimer, celles qui veulent détruire et haïr. Sont-elles contraires ou complémentaires? L'homme peut-il se passer de la seconde ? La première peut-elle l'emporter sur la seconde ? Y a-t-il des limites à l'éducabilité de l'homme ? Telles étaient les questions posées par Einstein.

La guerre pourrait-elle disparaître? "Tout en reconnaissant la nécessité biologique et psychologique de la souffrance pour l'économie de la vie humaine, on n'en a pas moins le droit de condamner la guerre dans ses moyens et ses buts et d'aspirer à la cessation des guerres ${ }^{109}$. " Que peut-on faire pour détourner des hommes la fatalité de la guerre ? À cette question, le premier réflexe de Freud est de se déclarer incompétent. Puis il entend «indiquer simplement comment le problème de la prévention des guerres se présente d'un point de vue psychologique ${ }^{110}{ }$. Il s'appuie sur la lettre d'Einstein, qui commence par les relations du droit avec le pouvoir. Il remplace le mot pouvoir par celui de violence : «le droit et la violence sont à nos yeux antinomiques », pourtant «il est facile de démontrer que l'un s'est développé à partir de l'autre, et si nous remontons aux origines, nous parvenons sans peine à la solution du problème ${ }^{111} »$. Freud part des conflits d'intérêt: ceux-ci sont fondamentalement tranchés par le recours à la violence. «Il en est ainsi dans tout le règne animal, dont l'homme ne devrait pas s'exclure. " Chez l'homme, s'ajoutent les conflits d'opinion; "mais c'est là une complication ultérieure ${ }^{112}$ ".

108. Des États créent une autorité pour arbitrer leurs conflit, même si une telle autorité doit trouver sa légitimité.

109. "Considérations actuelles sur la guerre et la mort ", p. 10.

110. «Pourquoi la guerre?» (1933), in Résultats, idées, problèmes II, p. 204.

111. Idem, p. 204.

112. Idem, p. 204. 
Dans la horde originaire, c'est la supériorité musculaire qui l'emporte: elle décide qui doit s'approprier quelque chose ou qui doit réaliser sa volonté. La force musculaire est bientôt augmentée et remplacée par l'utilisation d'outils. Avec l'introduction de l'arme, la supériorité intellectuelle commence à supplanter la force musculaire. L'intention ultime du combat reste la même: une partie doit être contrainte à abandonner ses prétentions ou son opposition. Ce but trouve sa réalisation la plus radicale lorsque l'adversaire est tué. L'avantage est double: il ne peut reprendre les hostilités; son sort dissuade les autres de suivre son exemple. «Par ailleurs, la mise à mort de l'ennemi satisfait un penchant pulsionnel ${ }^{113}$. » Des considérations utilitaires peuvent se substituer à l'élimination physique: dans ce cas, la violence se contente de soumettre l'adversaire au lieu de le tuer. C'est le début de la clémence. Mais le vainqueur doit alors compter avec la vindicte du vaincu. Comment est-on passé de la violence au droit?

La plus grande force de l'un pouvait être compensée par l'union de plusieurs faiblesses. La force de cette union représente le droit, par opposition à la violence d'un seul. En style nietzschéen : le droit, c'est le droit des faibles. Cette transition de la violence au droit suppose une condition : que la réunion des faibles soit stable et durable. La communauté doit élaborer des prescriptions qui préviennent les menaces de rébellion, désigner des instances qui veillent à l'observance des prescriptions et qui se chargent de l'exécution des actes coercitifs légaux. La reconnaissance de la communauté d'intérêt au respect du droit favorise des liens affectifs. L'essentiel est ainsi posé, en termes quasi hobbésiens ${ }^{114}$ : «La victoire remportée sur la violence par le transfert du pouvoir à une plus vaste unité dont la cohésion est maintenue par les liens affectifs entre ses membres. Tout le reste n'est que développements et répétitions ${ }^{115}$. » Les choses sont simples tant que la communauté est composée d'individus de force égale. Mais un tel état d'équilibre n'est concevable qu'en théorie. En réalité, la situation se complique du fait que la communauté englobe des éléments inégaux, hommes et femmes, parents et enfants, et bientôt, à la suite de guerres et de sujétions, vainqueurs et vaincus qui se transforment en maîtres et esclaves. Le droit de la communauté devient l'expression des rapports de force inégale entre gouvernants et gouvernés. Dès lors, il y a dans la communauté deux sources de désordre mais aussi d'évolution: les tentatives chez les dominants passer outre les limitations valables pour tous; les efforts chez les dominés changer les rapports de pouvoir. Les unes vont dans le sens de l'inégalité, les autres, dans celui de l'égalité. Lorsqu'elle n'est pas

113. Idem, p. 205.

114. "Le danger égal pour tous qu'est l'insécurité de la vie unit les hommes en une société qui interdit à l'individu la mise à mort et qui se réserve le droit de mettre à mort collectivement celui qui transgresse l'interdit. Cela s'appelle alors justice et punition " (L'avenir d'une illusion, p. 41). L'édifice de la civilisation repose ainsi sur le transfert à l'État du monopole de la violence, gérant l'agressivité à l'échelle collective : l'État hérite de l'omnipotence du père originel.

115. "Pourquoi la guerre?", p. 206. 
pacifique, la modification du droit en vigueur passe par la guerre civile, c'est-à-dire par la suspension du droit. Au sein même d'une communauté, le réglement des conflits d'intérêts par la violence n'est donc pas nécessairement évité : la violence n'est pas écartée entre compatriotes. Mais les solidarités et les besoins qui découlent de la cohabitation dans un même territoire facilitent la conclusion plus rapide de telles luttes.

L'histoire de l'humanité montre en revanche une série ininterrompue de conflits entre communautés qui sont tranchés par l'épreuve de force d'une guerre. De telles guerres se terminent soit par le pillage soit par la sujétion de l'une des parties. Les conquêtes ne relèvent pas d'un jugement uniforme : certaines n'apportent que des destructions, d'autres des bienfaits, ainsi de la pax romana. "Aussi paradoxal qu'il y paraisse, il faut avouer que la guerre ne serait pas un moyen inadapté pour établir cette paix 'éternelle' ardemment désirée, car elle est en mesure de créer ces vastes unités au sein desquelles un pouvoir central fort rend des guerres ultérieures impossibles ${ }^{116}$. " Mais les conquêtes ne sont pas durables, ou ne créent que des unions partielles... À l'époque présente, « une prévention efficace des guerres n'est possible que si les hommes s'entendent pour mettre en place un pouvoir central auquel est transféré le droit de jurisprudence pour tous conflits d'intérêts ${ }^{117}$ ». Se trouvent donc réunies deux exigences: qu'une instance suprême soit créée, qu'on lui confère le pouvoir nécessaire. Depuis 1919, une Société des Nations a été créée; elle n'a pas de pouvoir propre et ne peut l'acquérir que si les membres de la Société le lui cèdent. Deux éléments assurent la cohésion d'une communauté : la contrainte, les liens affectifs; la force, les intérêts communs. "C'est une erreur de ne pas considérer que le droit n'était à l'origine que violence et qu'il ne peut de nos jours non plus se passer du soutien de la violence ${ }^{118}$."

Quant à l'enthousiasme guerrier, il s'explique, selon Einstein, et Freud acquiesce partiellement, par la pulsion de destruction. Il y a en effet chez l'homme deux sortes de pulsions, aussi indispensables l'une que l'autre: celle de vie et d'amour, celle de mort et de haine. Il est rare que telle action soit l'œuvre d'une seule motion pulsionnelle. Ainsi, lorsque les hommes sont poussés à la guerre, les deux sortes de pulsions jouent, celle qui consiste à tuer l'ennemi («destruction»), mais aussi celle qui consiste à risquer sa vie pour la communauté ( «amour »). Tout homme recèle une pulsion de mort susceptible de se déchaîner.

Les bolcheviks espèrent faire disparaitre l'agression humaine en garantissant la satisfaction des biens matériels et en établissant l'égalité entre les membres de la communauté. Je tiens cela pour une illusion. Pour le moment, ils ont pris toutes les précautions pour s'armer, et la haine contre tous ceux qui sont à l'extérieur n'est pas leur moindre expédient pour maintenir la cohésion de leurs partisans ${ }^{119}$.

116. Idem, p. 207.

117. Idem, p. 208.

118. Idem, p. 209.

119. Idem, p. 212. 
Dans la démocratie développée, l'homme conserve donc son agressivité, c'est-à-dire la possibilité biologique et neurologique de la violence contre autrui. Ce qu'il s'agit de tenter, c'est de détourner cette agressivité suffisamment pour qu'elle ne trouve pas à s'exprimer dans et par la guerre.

À partir de notre doctrine mythologique des pulsions, nous trouvons une formule pour définir les voies indirectes de lutte contre la guerre. Si la propension à la guerre découle de la pulsion de destruction, on est tenté d'invoquer contre elle la pulsion antagoniste, l'Éros. Tout ce qui établit des liens affectifs entre les hommes ne peut que s'opposer à la guerre ${ }^{120}$.

Ces liens peuvent être de deux genres : l'amour, l'identification. "Aime ton prochain comme toi-même. » Voilà la formule - chrétienne - que Freud retient ${ }^{121}$. Contre la guerre, il propose donc le retour au christianisme originel, celui d'avant les doctrines médiévales de la guerre juste et de la guerre sainte. Mais l'abus d'autorité est une seconde invitation à lutter contre la guerre. La division entre gouvernants et gouvernés fait partie de l'inégalité des hommes. «L'État idéal serait une communauté d'hommes qui auraient soumis leur vie pulsionnelle à la dictature de la raison. Mais, selon tout vraisemblance, c'est là une espérance utopique ${ }^{122}$."

La psychologie, selon Freud, n'apporte finalement guère de solution pratique immédiate au problème de la prévention ou de l'élimination de la guerre. Il soulève une dernière question, qu'Einstein n'a pas évoqué dans sa lettre, mais qui requiert particulièrement son intérêt. Pourquoi nous révoltonsnous contre la guerre? "Elle semble pourtant conforme à la nature ${ }^{123}$." Pourquoi ne l'acceptons-nous pas? "La réponse sera sans doute que tout homme a un droit sur sa propre vie. » "C'est aussi que la guerre, sous sa forme actuelle, ne donne plus l'occasion de réaliser le vieil idéal héroïque ${ }^{124}$. $»$ Ces deux points sont cependant sujets à discussion. "On peut se demander si la communauté n'a pas non plus un droit sur la vie de l'individu; on ne peut condamner au même degré tous les types de guerre ${ }^{125}$.» La raison fondamentale de notre révolte contre la guerre est que nous sommes pacifistes. Et nous sommes pacifistes parce que, nous, civilisés, avons pleinement connu le processus de civilisation de l'humanité.

C'est à ce processus que nous devons le meilleur de ce que nous sommes devenus et une bonne partie des maux dont nous souffrons. Ses motifs et ses origines sont obscurs, son issue incertaine, certains de ses caractères aisément repérables. Peut-être mène-t-il à l'extinction de l'espèce humaine, car il porte atteinte à la fonction sexuelle à plus d'un égard, et dès aujourd'hui les races

120. Idem, p. 212.

121. Idem, p. 212.

122. Idem, p. 213.

123. Idem, p. 213.

124. Idem, pp. 213-214.

125. Idem, p. 214. 
incultes et les couches arriérées de la population s'accroissent plus fortement que celles qui sont très cultivées. Peut-être ce processus est-il comparable à la domestication de certaines espèces animales ${ }^{126}$.

Des modifications psychiques, sinon physiologiques, accompagnent le processus culturel : elles consistent en un déplacement des buts pulsionnels et en une limitation des motions pulsionnelles.

Parmi les caractères psychologiques de la culture, deux semblent les plus importants : le renforcement de l'intellect qui commence à dominer la vie pulsionnelle, l'intériorisation de la tendance à l'agression. Or les positions psychiques que le processus culturel nous impose se voient opposer par la guerre un démenti ; c'est pourquoi nous ne la tolérons plus. [Mais] combien de temps devrons-nous attendre avant que les autres eux aussi deviennent pacifistes ? On ne saurait le dire, mais peut-être n'est-ce point un espoir utopique de penser que l'influence de ces deux facteurs, celui des positions culturelles et celui de la crainte justifiée d'une guerre future, mettra fin aux entreprises guerrières dans un avenir peu éloigné. Par quel tour ou détour, nous ne pouvons l'augurer. En attendant, il nous est permis de nous dire : tout ce qui promeut le développement culturel œuvre du même coup contre la guerre ${ }^{127}$.

En conclusion, Freud mise donc sur les mentalités, sur la «civilisation des mœurs » (N. Elias), pas sur les institutions : ni sur le régime politique (la démocratie) ni sur le droit international (la SDN), sauf dans la mesure où ceuxci reflètent une évolution des mentalités. Il attend d'autant moins de la démocratie, il est d'autant moins kantien qu'il estime que les décideurs doivent être indépendants des masses :

Les masses n'aiment pas le renoncement pulsionnel, et les individus qui les composent se confortent mutuellement en donnant libre cours à leur dérèglement. Seule l'influence d'individus exemplaires, qu'ils reconnaissent comme leurs meneurs, peut les amener à des renonciations dont dépend l'existence de la culture. Tout va bien lorsque ces meneurs sont des personnes qui se sont hissées jusqu'à la domination de leurs propres souhaits pulsionnels. Mais ils courent le danger, pour ne pas perdre leur influence, de céder à la masse plus que celleci ne leur cède, et c'est pourquoi il apparaît nécessaire qu'ils disposent de moyens de puissance pour être indépendants de la masse ${ }^{128}$.

C'est dans l'autorité, raisonnable et sans abus, que réside donc la chance de la paix.

126. Idem, p. 214.

127. Idem, p. 215.

128. L'avenir d'une illusion, p. 8. 


\section{Bibliographie}

Sigmund Freud présenté par lui-même (1925, 1935), Paris, Gallimard, 1984.

Freud, Sigmund. Choix de textes, rassemblés par Th. Laveyssière, Paris, Masson, 1991, "Bibliographie ", pp. 5-17, "Biographie", pp. 18-27.

Freud, Sigmund. Totem et tabou (1913), Paris, Petite Bibliothèque Payot, 2001.

- "Considérations actuelles sur la guerre et sur la mort» (1915), in Essais de psychanalyse, Paris, Payot, 1981, pp. 7-40.

—. "Psychologie des foules et analyse du moi " (1921), in Essais de psychanalyse, pp. 123-217, notamment le chapitre sur l'Église et l'Armée, pp. 153-160.

- L'avenir d'une illusion (1927), Paris, Quadrige/PUF, 1995.

- Le malaise dans la culture (1930), Paris, Quadrige/PUF, 1998. . « Pourquoi la guerre ?» (1933), in Résultats, idées, problèmes II, 1921-1938, Paris, PUF, 1985, pp. 203-215.

- Le Président Thomas Woodrow Wilson. Portrait psychologique (1932-1938), avec la collaboration de William C. Bullitt, Paris, A. Michel, 1967.

- L'homme Moïse et la religion monothéiste. Trois essais (1934-1938). Paris, Gallimard, 1986.

Chartier, Jean-Pierre (1993). Introduction à la pensée freudienne. Les concepts fondamentaux de la psychanalyse, Paris, Petite Bibliothèque Payot, 2001.

Chemouni, Jacquy. "Psychanalyse et politique : 1900-1940", in J. Vanwelkenhuyzen (dir.): Les tumultes d'un siècle, Bruxelles, Complexe, 2000, pp. 131-156.

Glaser, Hermann. Sigmund Freud et l'âme du XXème siècle, Paris, PUF, 1995.

Raulet, Gérard. «La mort aux deux visages. Sur le statut de l'agressivité et de la pulsion de mort dans Malaise dans la civilisation, in Jacques Le Rider, Michel Plon, Gérard Raulet, Henri Rey-Flaud: Autour du «Malaise dans la culture » de Freud, Paris, PUF, 1998, pp. 55-78. 